\title{
Efficacy of compartmentalization in controlling an adenovirus type 54 keratoconjunctivitis outbreak on Oki Island, Japan
}

\author{
Yutaka Omatsu ${ }^{1} \cdot$ Dai Miyazaki $^{1} \cdot$ Yumiko Shimizu $^{1} \cdot$ Kazuki Matsuura $^{2} \cdot$ Shin-ichi Sasaki ${ }^{1} \cdot$ Yoshitsugu Inoue $^{1}$. \\ Eiichi Uchio ${ }^{3}$. Tsuguto Fujimoto ${ }^{4}$
}

Received: 11 March 2020 / Accepted: 9 December 2020 / Published online: 25 February 2021

(c) Japanese Ophthalmological Society 2021

\begin{abstract}
Purpose To analyze the epidemiologic characteristics of an outbreak of human adenovirus type 54 (HAdV-54) on Oki Island, Shimane Prefecture, Japan, in 2017 and to assess the effectiveness of a compartmentalization method in controlling the incidence and spread.

Study design Retrospective cohort study.

Methods The infection was diagnosed in 136 individuals, and typing was confirmed by PCR and direct sequencing. The epidemiologic characteristics of the disease including the infection rate, incubation period, and basic reproductive number $\left(\mathrm{R}_{0}\right)$, ie, number of cases directly infected by an infectious patient during the course of the disease, were investigated. The effectiveness of compartmentalization for infection control was determined by simulating the outbreak using the SusceptibleExposed-Infectious-Recovered (SEIR) model.

Results The majority of the HAdV-54-infected individuals were the children of 3 nursery schools (A, B, and C) and their parents on Oki Island. The infection rates in the 3 schools were $13.2 \%, 16.9 \%$, and $17.2 \%$, respectively. The one class of school B without the index case was initially compartmentalized, and the infection rate in this compartment was $0 \%$. The incubation period was calculated to be $9.3 \pm 3.5$ days, and the disease duration, $13.0 \pm 5.4$ days. The $\mathrm{R}_{0}$ was 1.43 . Using these parameters, a SEIR model was constructed. The SEIR model well predicted the daily incidence of infection and indicated that the compartmentalization method provides effective reduction in the incidence of the infection, with much earlier control.

Conclusions The compartmentalization method is effective to control HAdV-54 outbreaks.
\end{abstract}

Keywords Adenovirus type 54 - Basic reproductive number - Compartmentalization - Epidemic keratoconjunctivitis · Susceptible-exposed-infectious-recovered model

\section{Introduction}

Corresponding Author: Yutaka Omatsu

Yutaka Omatsu

yutakaOMT@gmail.com

1 Division of Ophthalmology and Visual Science, Faculty of Medicine, Tottori University, 36-1 Nishi-cho, Yonago Tottori 683-8504, Japan

2 Division of Ophthalmology of Nojima Hospital, Tottori, Japan

3 Department of Ophthalmology, Faculty of Medicine, Fukuoka University, Fukuoka, Japan

4 National Institute of Infectious Diseases, Tokyo, Japan
Human adenovirus (HAdV) is a major pathogen that can cause acute viral keratoconjunctivitis, which manifests clinically as epidemic keratoconjunctivitis (EKC) [1]. HAdV spreads through direct contact and maintains its infectivity for up to 3 weeks $[1,2]$. Filamentous keratitis and corneal erosion and ulcers can develop in the severe forms of EKC. These complications are followed by multiple subepithelial corneal infiltrates (MSI), which can cause a reduction in vision for several months or years [3, 4].

Members of the HAdV D species have been the main cause of EKC outbreaks, eg, outbreaks in Asia, the United States, and Europe [5]. HAdV type 8 (HAdV-8) is the major type and accounted for $83 \%$ of HAdV species D infections as calculated by reports from the United States, India, Brazil, 
and Sri Lanka [5]. Recently, HAdV type 54 (HAdV-54), genetically close to HAdV-8, has become the dominant type in Japan [6-9]. Since it was first reported in 2000 [6], 70.4\% of HAdV-positive cases were typed as HAdV-54 in Osaka, Japan in 2016 [10]. HAdV-54 has emerged as a significant pathogen with a possible worldwide spread.

In 2017, an outbreak of HAdV-54 occurred on Oki Island, a small isolated island of Shimane Prefecture in the Sea of Japan. The number of EKC patients amounted to 136 at the end of the outbreak. The population of Oki Island is approximately 14,000 , and the final infection rate reached $0.97 \%$ of the island population.

In this isolated environment, the outbreak was managed by only 1 ophthalmologist in only 1 hospital on Oki Island. This allowed detailed analysis of the outbreak pattern, which is often hampered by many kinds of personal interaction involving many hospitals. Under these conditions, we analyzed how HAdV-54 spread and analyzed the characteristics of the HAdV-54 outbreak.

The purpose of this report is to present our findings on the treatment protocols and their results in reducing the incidence of HAdV-54 and bringing it under control. To accomplish this, we examined the effects of compartmentalization of the cohorts and the pattern of the spread of HAdV-54. We determined the risk factors using the Susceptible-ExposedInfectious-Recovered (SEIR) model. The SEIR model is determined on the basis of the basic reproductive number $\left(\mathrm{R}_{0}\right)$, disease duration, and incubation period. Thus, this model allows easy simulation of virus infections when these parameters have been determined. Our data provide essential information on building and instituting effective infection control for HAdV-54 outbreaks.

a

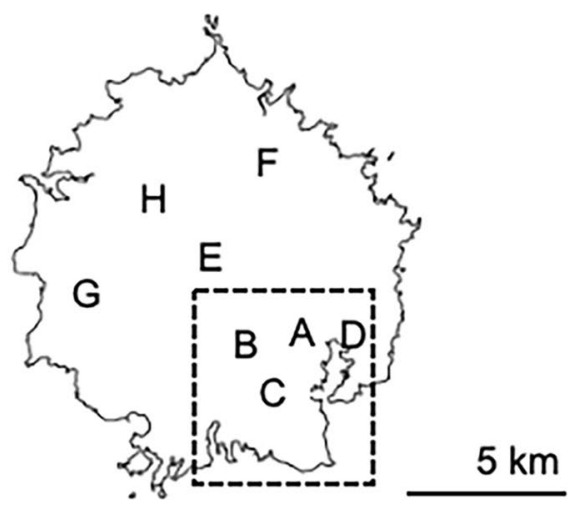

Fig. 1 Demographics of the outbreak of adenovirus type 54 keratoconjunctivitis on Oki Island in Shimane Prefecture, Japan. a Map of the locations of 8 nursery schools on Oki Island. The square area surrounded by the broken line is the area of map $\mathbf{b}$. $\mathbf{b}$ The circled area

\section{Methods}

\section{Epidemiologic investigation of the adenovirus outbreak}

The Oki Islands are volcanic in origin and comprise more than 180 islands including small inhabited islands. In September 2017, the index case of EKC was identified to be an outpatient at Oki Hospital in Dogo, the main island of the Oki Islands chain. There are 8 nursery schools (A, B, C, D, E, F, G, and H) in Dogo (Fig. 1a). Schools A, B, C, and $\mathrm{D}$ were located within a $2-\mathrm{km}$ radius of the central district centered on Saigo Port (Fig. 1b). There were 76, 172, 64, 34, $32,24,22$, and 18 students in schools A, B, C, D, E, F, G, and $\mathrm{H}$, respectively. The outbreak was observed in 3 nursery schools (A, B, and C).

EKC was diagnosed in the initial patient by use of an immunochromatography kit for HAdV (Capilia Adeno Eye; Tauns Laboratories, Inc). This case was followed by an increase in the number of acute conjunctivitis cases in the outpatient clinic of Oki Hospital. The HAdV outbreak was first observed in school B and spread to schools A and C.

The Oki Hospital is the only hospital on Dogo, and it provides complete eye care services. All the EKC patients on the island were diagnosed and followed by the 1 ophthalmologist (Y.O.) at Oki Hospital. The diagnosis of EKC in the outbreak cases was made according to the clinical symptoms and confirmed by positivity to HAdV shown by immunochromatography. Immunochromatography testing was conducted for 48 of the 136 cases. The clinical diagnosis was made according to intrafamily spread and the characteristics of the clinical course of EKC.

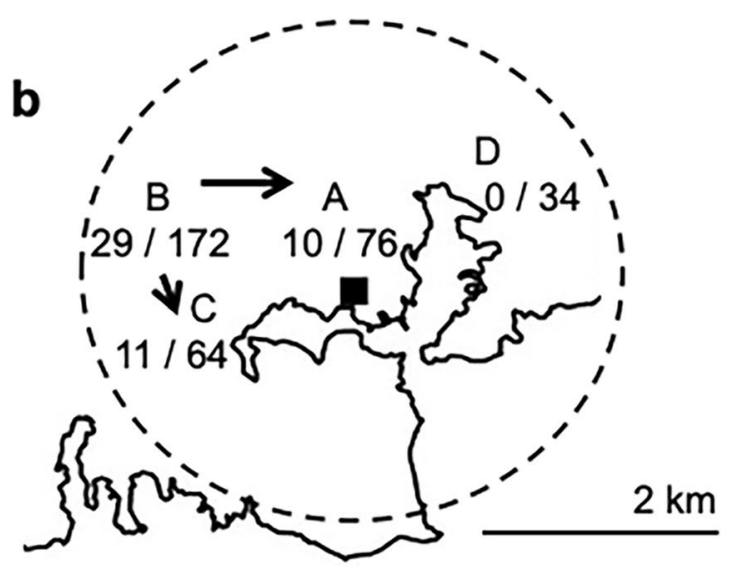

within the broken line is the area within $2 \mathrm{~km}$ of Saigo Port (filled square). There are 4 Schools, A, B, C and D, in the circled area. The numbers indicate the infected/total number of children. The arrow indicates the infection route and outbreak spread from school B 
The incubation period was calculated on the basis of the mean period between the first and second infections in a family, assuming that intrafamily contact caused the infection of the other family members.

Our epidemiologic investigation included a review of the medical records of the infected individuals and interviews with the staff of the nursery schools. The demographic characteristics were examined to identify how the outbreak spread among the students and parents. This study was approved by the ethics committee of Oki Hospital.

\section{Modeling of the outbreak}

To model the outbreak, we calculated the basic reproductive number $\left(\mathrm{R}_{0}\right)$, which is the number of individuals directly infected by 1 infectious individual in a susceptible population during the entire infectious period. The $\mathrm{R}_{0}$ indicates the number of infections generated by 1 case in a cohort where all the individuals are susceptible to infection: $R_{0}=\beta / \gamma$, where the preintervention transmission rate is $\beta$ and the mean infectious period is $1 / \gamma[11]$. On the basis of these values, the outbreak was mathematically analyzed using the SEIR model assuming random contacts in a compartmental spread in the nursery schools. The SEIR model consisted of 4 compartments: the susceptible (S), exposed (E), infectious (I), and recovered (R) individuals. The number of individuals in the 4 compartments varied over time and was therefore calculated as a function of time. The SEIR model is defined as

$\frac{d S}{d t}=-\mu S-\frac{\beta S I}{N}$

$\frac{d E}{d t}=\frac{\beta S I}{N}-(\mu+a) N$

$\frac{d I}{d t}=a E-(\gamma+\mu) I$

$\frac{d R}{d t}=\gamma I-\mu R$

where the incubation period is $1 / a$, the death rate is $\mu$, and $N=S+E+I+R$ (Fig. 4a) [11]. For simulation of the SEIR model, the python program code is provided as Online Resource 1.

\section{Management of the adenovirus outbreak}

To prevent the spread of EKC, the patients and their family members with EKC were instructed to wash their hands and not to share daily necessities. They were also instructed to stay at home and were confined there for more than 9 days from the disease onset or until they became asymptomatic. Asymptomatic family members were instructed to avoid contact with infected patients.

Levofloxacin $(0.5 \%)$ eye drops were used to treat all the patients. Steroid eye drops $(0.1 \%$ fluorometholone or betamethasone) were also used when the clinical signs were severe.

To prevent in-hospital infection, clinical examinations of suspected patients were conducted in a different room using different appointment times. An EKC alert was posted in the major district of the island including the hospital to notify visitors or patients of the outbreak of EKC. For the Oki islanders, an island broadcast system using loudspeakers was used to alert them of the outbreak and provide information on EKC.

For compartmentalization, the class of 5-year-olds (B1 class) $(n=30)$ of school $B$, which had no direct contact with infected children, was compartmentalized from the other class (B2 class) $(n=142)$ in school B and the other schools at the earliest stage of outbreak. The B2 class of school B, which included asymptomatic children, was compartmentalized before a second wave of infection occurred. Schools A and $\mathrm{C}$, where outbreaks occurred, were also managed as separate compartments, and schools $\mathrm{D}, \mathrm{E}, \mathrm{F}, \mathrm{G}$, and $\mathrm{H}$, where no infected child was found at the end of the outbreak, were also managed as separate compartments. Schools A, B, C, and D were located close to each other in the downtown district of Oki. For compartmentalization, the children, parents, teachers, and school employees were instructed not to interact with individuals belonging to the other school compartments until the outbreak ended.

\section{PCR typing of adenovirus infection}

For the typing of adenovirus, 8 representative cases were chosen on the basis of the transmission route of infection in the nursery schools. Adenovirus typing was conducted by use of PCR, and direct sequencing following the protocol developed by the National Institute of Infectious Diseases [12]. Conjunctival swab samples were stored frozen, and DNA was extracted by use of the QIAamp DNA mini kit (Qiagen). The extracted DNA was amplified using PrimeSTAR GXL DNA polymerase (Takara) with the following primers targeting the adenovirus: hexon loop 1: forward (5'CACATCGCCGGACAGGATGCTTCGGAGTA-3'), reverse (5'-GTGTTGTGAGCCATGGGGAAGAAGGTGGC-3') [13]; fiber code: forward (5'-GATGTCAAATTCCTGGTC CAC- $3^{\prime}$ ), reverse (5'-TACCCGTGCTGGTGTAAAAATC3') [14]; penton base: forward (5'-CARAAYGAYCACAGC AACTT-3'), reverse (5'-GCRGGMACGTTTTCACTR ACGGT-3') [15]. The amplification reaction was carried out with preliminary denaturation at $94{ }^{\circ} \mathrm{C}$ for $5 \mathrm{~min}$, followed by 30 cycles of denaturation at $98^{\circ} \mathrm{C}$ for $30 \mathrm{~s}$, annealing at 
$57{ }^{\circ} \mathrm{C}$ for $30 \mathrm{~s}$, and primer extension at $68^{\circ} \mathrm{C}$ for $2 \mathrm{~min}$ for hexon loop1. For the fiber code and penton base, 40 cycles of denaturation at $98^{\circ} \mathrm{C}$ for $30 \mathrm{~s}$, annealing at $55^{\circ} \mathrm{C}$ for $30 \mathrm{~s}$, and primer extension at $68^{\circ} \mathrm{C}$ for 2 min were used. The amplified products were directly sequenced. The resulting sequences were used to identify the adenovirus type by use of the basic local alignment search tool (BLAST).

\section{Statistical analyses}

Data were expressed as means (SDs). The disease duration was defined as the duration until the date of complete resolution of the objective symptoms. The multivariate Cox proportional hazards model was used to calculate the hazard ratio of clinical characteristics on disease duration. Statistical analyses were conducted using Stata 15 software (StataCorp). Probability values below 0.05 were considered significant.

\section{Results}

The HAdV-54 outbreak occurred on the isolated island of Oki Island (Dogo) from September to December 2017. Of the 136 patients, $72(52.9 \%)$ were male. The age distribution of the infected individuals was bimodal, and the major infected populations were individuals aged younger than 6 years and their parents in their thirties (Fig. 2). Fiftyone patients $(37.5 \%)$ were aged $\leq 6$ years, and 50 of them belonged to nursery schools. For the nursery schools, the mean (SD) age was 2.7 (1.7) years, and the median age was 3 years, and the boys made up $58.0 \%$ of these patients $(n=29)$. The patients not belonging to the nursery schools were mainly their siblings or parents. The mean age of these patients was 36.1 (16.6) years, and the median age was 35 years. The percentage of infected boys and men was $49.4 \%(\mathrm{n}=42)$.

HAdV-54 was brought to Oki Island by a woman infected with HAdV-54 who had traveled to Oki Island from Ehime Prefecture, in mainland Japan. The index case on Oki Island was a 68 -year-old man who was infected by direct contact with the traveler. Then, HAdV-54 was transmitted to his 37-yearold son and 3 grandchildren belonging to school B $(n=172)$. The 3 children belonged to B2 class $(n=142)$ of school B, and B2 class was not suspended for more than a week. After the detection of EKC cases in B2 class, the children and staff of B1 class were strictly advised not to have any contact with members of B2 class and to compartmentalize. In B2 class, EKC was transmitted to other children; however, in B1 class, no one was infected during this outbreak. The spread to schools A and C subsequently occurred presumably by direct contact with infected children possibly at public playgrounds or supermarkets, which were used by most of the islanders in a
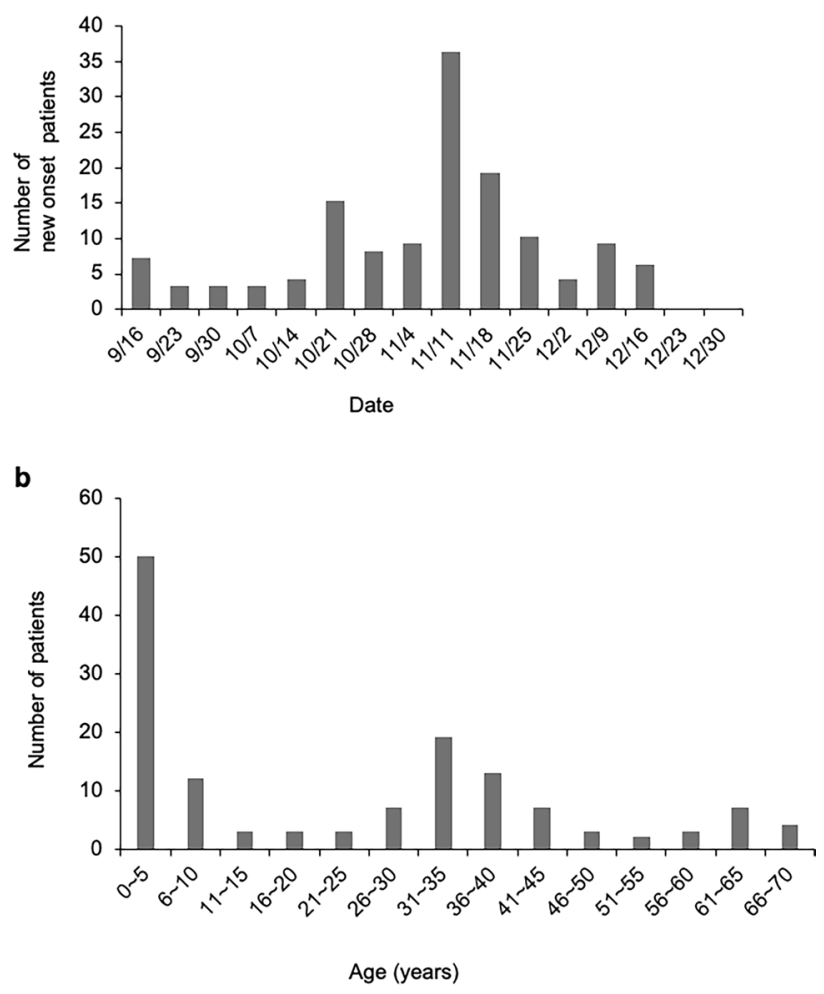

Fig. 2 Outbreak of adenovirus type 54 epidemic keratoconjunctivitis on Oki Island. a The number of newly infected individuals in the entire population peaked in early November 2017. b The age distribution was bimodal: $\leq 5$ and $30-35$ years

the neighborhood. Thus, the outbreak spread to schools A and C through siblings, family members, or friends of B2 class. The outbreak first spread within the schools and then to the families of the infected children. The cumulative infection rate in school A was $13.2 \%$; in school B, 16.9\%; and in school C, $17.2 \%$ (Fig. 3). There were 8, 17, and 13 cases of suspected family transmission from infected children in schools A, B, and $\mathrm{C}$, respectively. The mean number of days from the diagnosis of EKC in the child until that of the child's family members was 9.9 (6.3) days, with a range of 0 to 21 days.

The incubation period was calculated using family infection cases. The mean incubation period was 9.3 (3.5) days.

We also assessed how many individuals were directly infected by 1 infected person in the early period of this outbreak. The basic reproductive number $\left(\mathrm{R}_{0}\right)$ was calculated to be 1.43 .

\section{Effectiveness of compartmentalization for the schools}

At the end of the outbreak, no new patients were found who needed to be compartmentalized in B1 class of school B or in schools D, E, F, G, and H. 
Fig. 3 Time course changes of the patients, nursery school children and their family members. The outbreak began in school B, and then in schools $\mathrm{C}$ and $\mathrm{A}$. The blue bar indicates the number of nursery school children infected in the nursery schools. The red bar indicates the number of family members of the infected children of the nursery schools. The findings suggested that most members of the infected families were infected by children infected at the nursery schools a

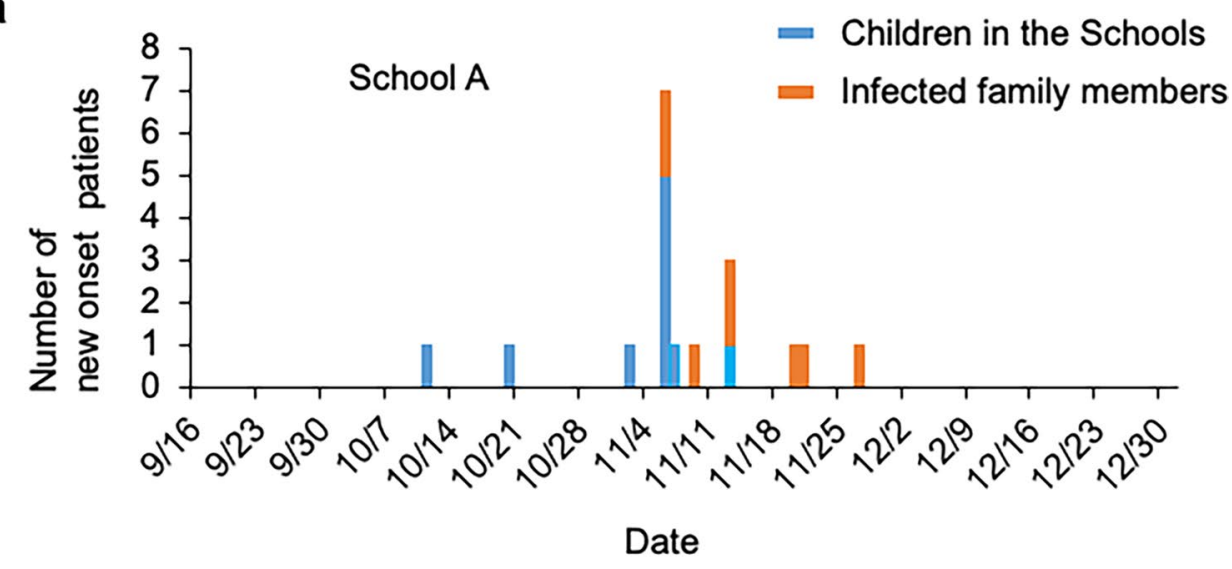

b

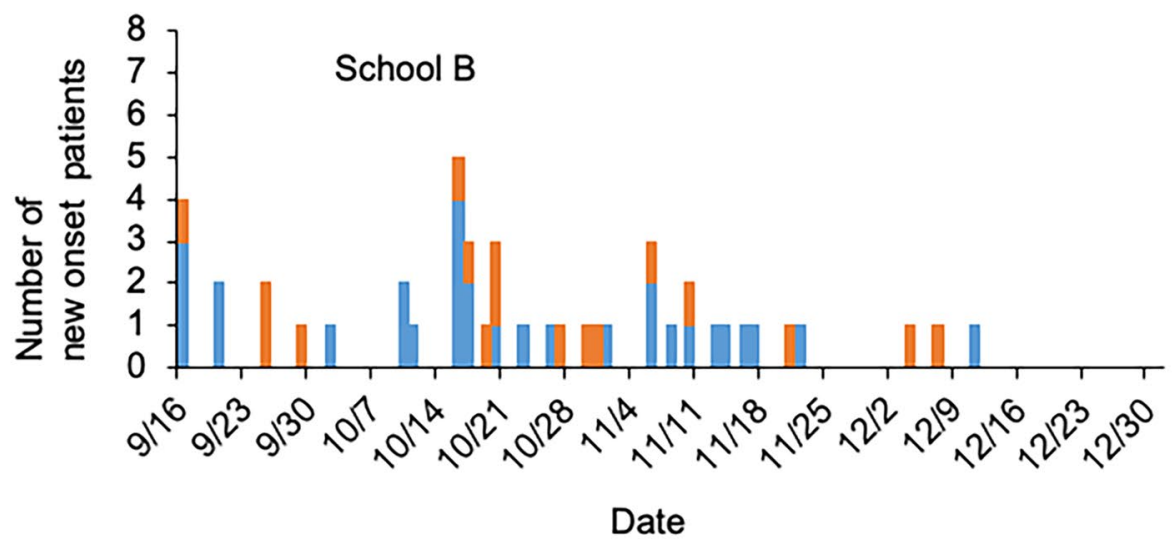

C

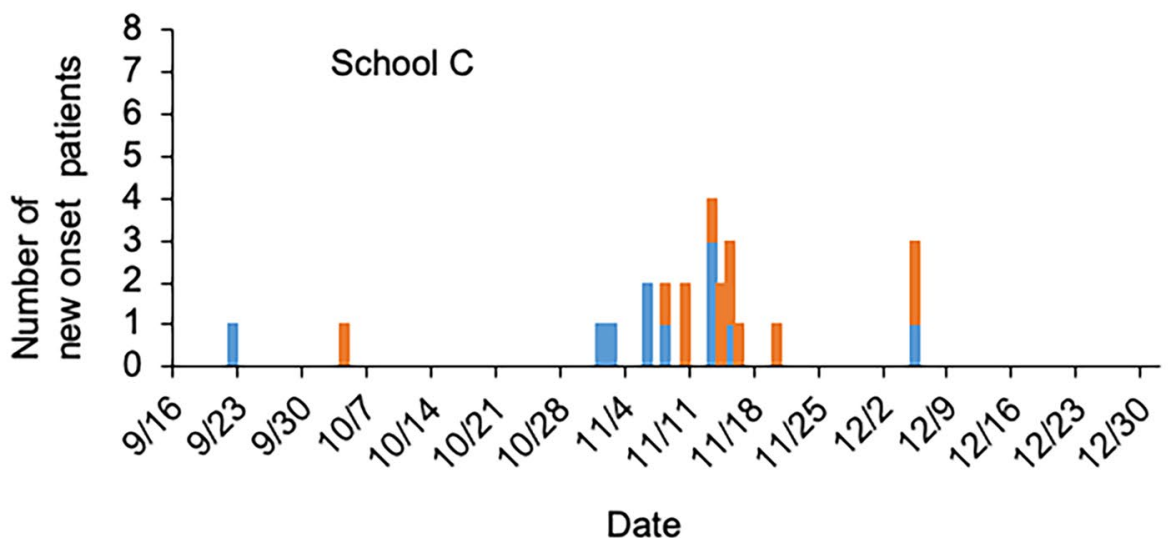

Because the schools were compartmentalized to prevent the spread of EKC, we applied the SEIR model to model the cumulative infection number during the time frame of the outbreak in school A, B2 class of school B, and school C (Fig. 4b-d). The SEIR model well simulated the increase in infection cases during this time frame. The results of the SEIR model indicated that the highest cumulative infection number was in B2 class of school B (Fig. 4c). This was specifically determined by the number of index cases, ie, the number of the first infected patients in each school, which was 3 in B2 class of school B and 1 each for schools A and C.

We applied compartmentalization of B2 class of school B using this SEIR model (Fig. 5a). When the B2 class was not compartmentalized, the cumulative infection rate was $20.9 \%$. In the scenario of 1 compartment with 1 exposed and the other with 2 exposed but asymptomatic children, the cumulative infection rate was similar (19.8\%). When B2 class was divided into 1 compartment with only unexposed children and the other with 3 exposed 
a

\begin{tabular}{|c|c|c|c|c|c|c|}
\hline & $\frac{\beta S I}{N}-a N$ & & $a E-\gamma I$ & & $\gamma I$ & \\
\hline Susceptible & $\longrightarrow$ & Exposed & $\longrightarrow$ & Infectious & $\longrightarrow$ & Recovered \\
\hline
\end{tabular}

b

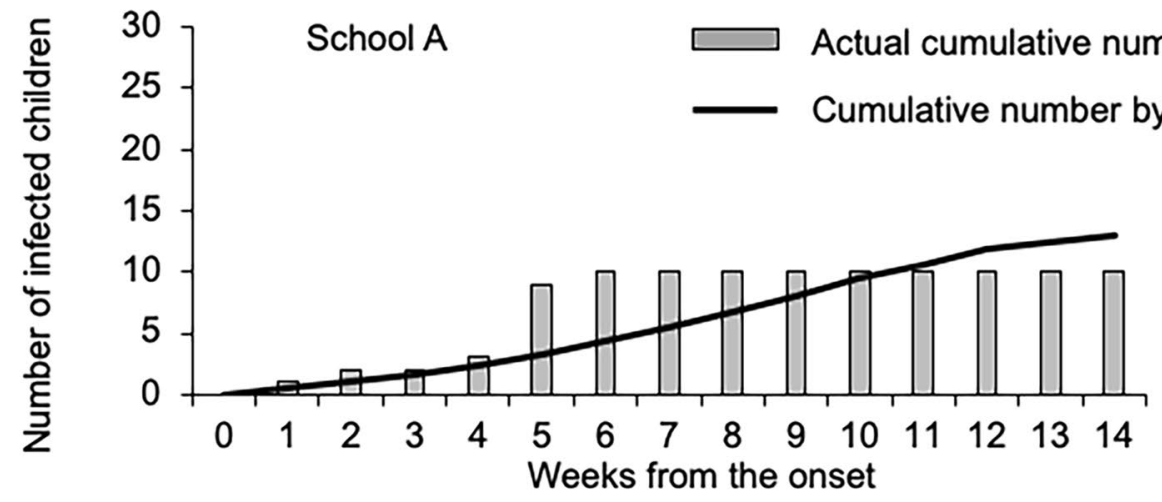

C

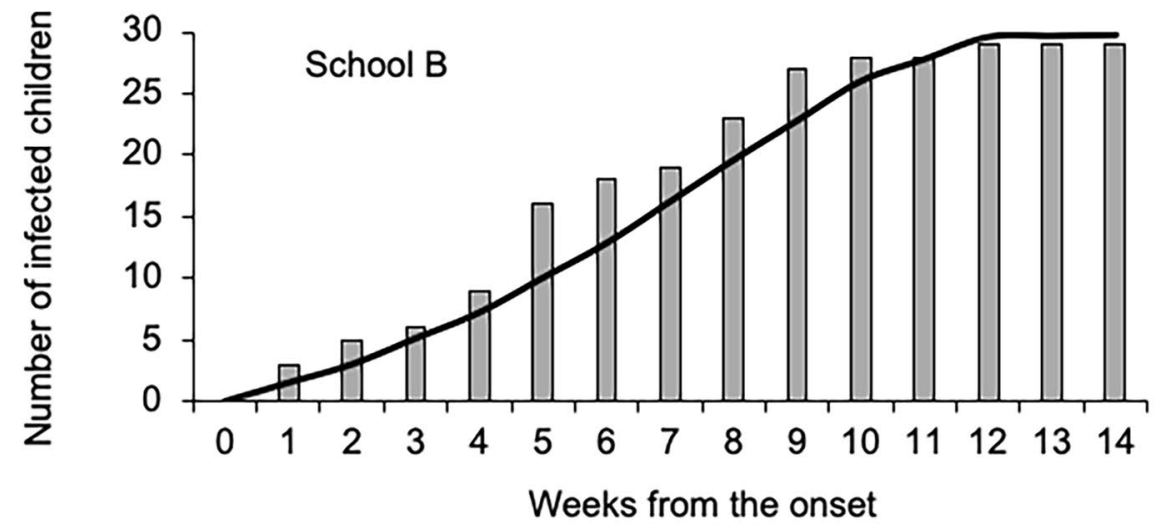

d

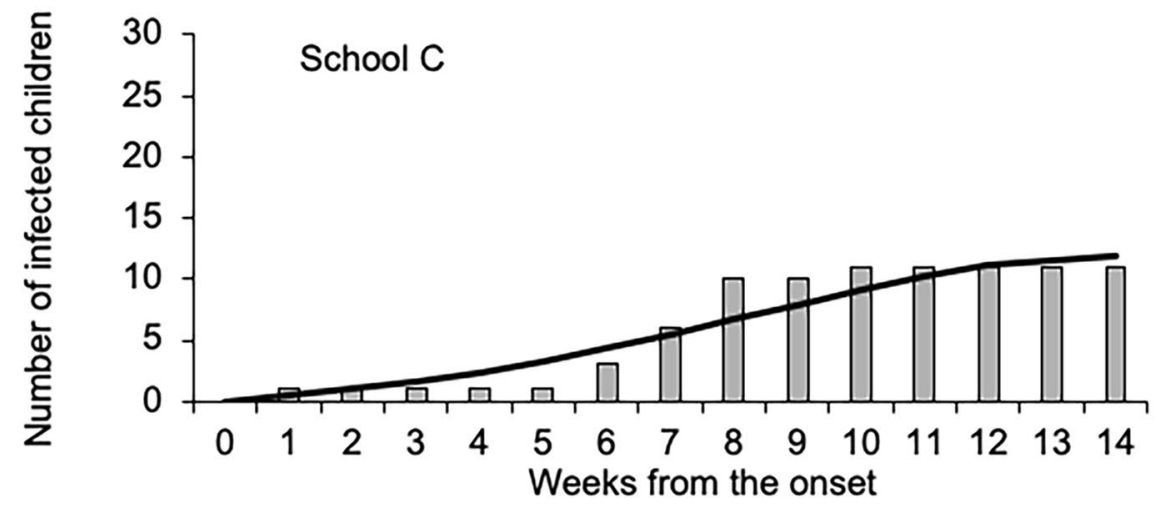

Fig. 4 Nursery school-based cumulative infection and prediction using the SEIR model. a Flow diagram of the SEIR model consisting of 4 compartments: the susceptible (S), exposed (E), infectious $(\mathrm{I})$, and recovered $(\mathrm{R})$ individuals. $\beta$ : preintervention transmission rate; $\gamma$ : $1 /$ mean infectious period; $a$ : 1 /mean incubation period. The 3 graphs below represent school A (b), B2 class of school B (c), and school C (d). The bar chart indicates the actual cumulative number of infections. The line chart indicates the predicted cumulative number of infections according to the SEIR model using an $\mathrm{R}_{0}$ of 1.43 and an incubation period of 9 days. The highest cumulative number of infections was observed for B2 class of school B (c), where the number of index cases was 3 , as compared with 1 each for schools $\mathrm{A}(\mathbf{b})$ and $\mathrm{C}$ (d) 


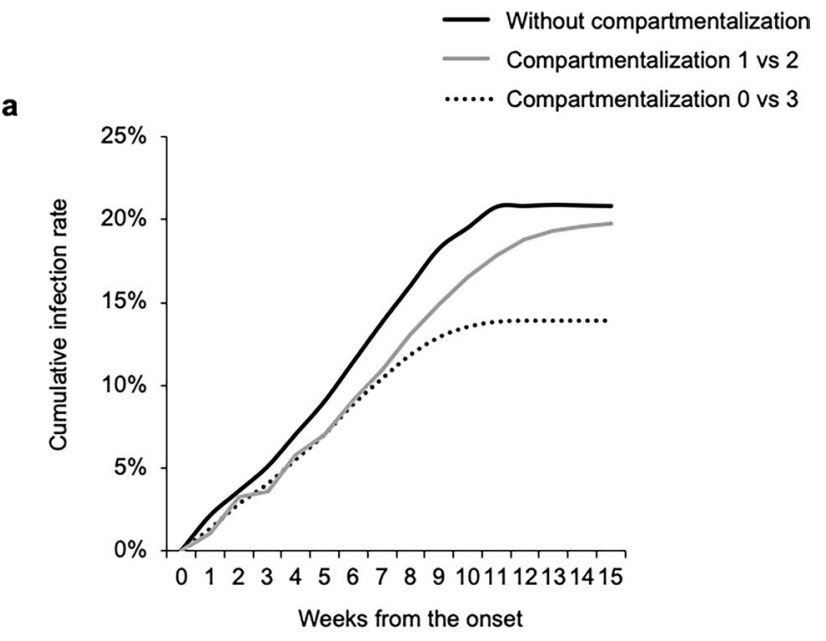

b

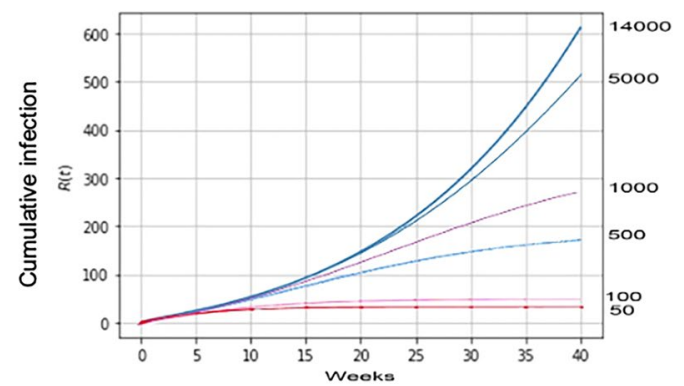

Fig. 5 Effect of compartmentalization on cumulative infection rate predicted by the SEIR model. a The cumulative infection rate of B2 class of school B was calculated using the SEIR model. The cumulative infection rate without compartmentalization was $20.9 \%$ (solid line). The gray line indicates the results in the case that B2 class of school B was compartmentalized for 1 compartment with 1 exposed and the other with 2 exposed but asymptomatic children. The dotted line indicates the effective compartmentalization by which B2 class of school B was divided into 1 compartment with only unexposed children and the other with 3 exposed but asymptomatic children. The cumulative infection rate was reduced to $13.9 \%$. b Simulation of cumulative infection in different compartments of 14,000 islanders by use of the SEIR model. The number of indices is set to 10 . The largest compartment was 14,000 . When the compartment size was reduced to $5000,1000,500,100$, and 50 , the cumulative number of infections was significantly reduced, and an earlier ending of infection (plateau) was observed. The ordinary differential equation of the SEIR model was solved using Online Resource 1 Code (txt file and ipynb file)

but asymptomatic children, the cumulative infection rate was reduced to $13.9 \%$.

We then simulated the effects of compartmentalization using the defined SEIR model on a hypothetical outbreak over the entire island (Fig. 5b, Online Resource 1 Code for the SEIR model). The onset of the infection in 10 infected patients in 14,000 islanders was simulated. When random movement or contact within the island was allowed after onset, 600 cases were observed after 40 weeks, and still the infection was not contained. The 10 infected cases were compartmentalized to a single compartment of 50-5000. When compartmentalization to 50 and no contact with the other islanders was used, the increase in the cumulative number of EKC patients become a plateau as earlier as 10 weeks, and the endemic ended. However, when a larger compartment, such as 1000 , was used, the plateau was delayed, and infection control was not achieved within 40 weeks. Thus, the SEIR model indicates that control of random movement or contact is an effective method to achieve earlier and effective control of an outbreak.

\section{Discussion}

HAdV outbreaks commonly occur in hospitals, nursery schools, and other level schools. Generally, the most effective method to end such outbreaks is a closure of the hospital wards and school classes. On Oki Island, the socioeconomical demands of working parents did not permit closure of the nursery schools. Our results showed the natural course of the HAdV-54 outbreak under the protocols of hand hygiene and social containment. We analyzed the HAdV-54 outbreak on a small isolated island and have assessed the effects of compartmentalization and our preventive measures to bring HAdV-54 under control.

The transmission potential determines the outcome, and it differs depending on the viral strain and can be expressed as the number of cases of EKC caused by 1 infected individual in a completely susceptible cohort over the course of the infectious period. This is the basic reproductive number, $\mathrm{R}_{0}$, which is determined by the specific virus and mode of infection. The high $\mathrm{R}_{0}$ for measles, which is transmitted by an airborne virus, is $10-20$. The $\mathrm{R}_{0}$ for HAdV-54 was calculated to be 1.43, and this value is similar to that for the Ebola virus of 1.36-1.49 [16] and for norovirus of approximately 2.0 [17]. For SARS-CoV-2, the estimate of $\mathrm{R}_{0}$ was reported to be 2.79 (median) [18].

Ebola virus and norovirus are transmitted by direct contact with body fluids, and they are nonenveloped and difficult to disinfect with alcohol. However, they have completely different outbreak patterns. Attempts to contain the Ebola virus in recent years has repeatedly failed, resulting in massive outbreaks in African countries. The main reason for this failure was determined to be a shortage of health and socioeconomic workers, lack of medical resources, lack of leadership or governance, and lack of communication [19].

Norovirus often causes nosocomial infections, and it spreads rapidly. Norovirus is environmentally stable and present at sufficient levels for infection [20, 21]. These relatively common characteristics and similar $\mathrm{R}_{0}$ values suggest a preventive strategy for norovirus may also be applied for HAdV infections. In hospital outbreaks of norovirus, early compartmentalization of the symptomatic patients was 
shown to effectively reduce the duration of the outbreak [22]. For HAdV infections, it is better for symptomatic individuals not to attend school. Therefore, we proposed that compartmentalization was needed for asymptomatic subjects before the development of any symptoms at the early stage of the outbreak.

The recent SARS-CoV-2 pandemic infection has spread mainly through respiratory droplets. Contaminated surface or aerosol spread is considered a possible mode of transmission [23]. Different from SARS-CoV, SARS-CoV-2 transmission can occur via presymptomatic carriers, and these comprise almost one-half of all transmissions [24]. Transmission of HAdV-54 by asymptomatic individuals is considered uncommon [25]; however, the mode of presymptomatic transmission is problematic for the prevention of spread. For SARS-CoV-2, compartmentalization may also become effective; however, this may require an emergency order because discrimination between presymptomatic infected and healthy individuals is difficult even with PCR testing.

We calculated the basic reproductive number, $\mathrm{R}_{0}$, of HAdV-54, which has not been previously reported. This $R_{0}$ value was determined for the time course and cumulative infection rate of each nursery school. $\mathrm{R}_{0}$ is the product of 3 factors: the probability of infection, number of contacts with infected individuals, and duration of the infectiousness. Thus, all of these factors are also important to bring the outbreak under control.

The probability of infection with HAdV can be reduced by infection control support including hand hygiene and use of disinfectants. For further control of an outbreak, a reduction in the number of contacts was effective (see Fig. 5). The major difficulty of early compartmentalization is how to accurately identify the exposed but asymptomatic subjects. In our cases, we identified individuals who had no opportunity for contact with EKC patients, and others considered exposed but asymptomatic thus needed to be contained. The SEIR model confirmed that the final cumulative infection rate was much lower and an earlier end of the outbreak was expected when proper compartmentalization was applied.

As is modeled by SEIR, the final cumulative infection rate is determined by $R_{0}$, which is the function of the infection rate and the infectious period. Therefore, we also evaluated the factors that were significantly associated with prolongation of the disease duration. Online Resource 2 (Supplementary Table 1) shows the demographic and clinical characteristics of HAdV-54 EKC. Using the Cox proportional hazards model, we found that the presence of MSI, which developed 19.0 (9.5 days) after the onset, and pseudomembranes prolonged the disease duration by 2 to 3 times (hazard ratio, 3.22; $P=0.005$; Online Resource 3 ). This is consistent with the findings of an earlier report [5]. The presence of MSI had the strongest effect on the prolonged duration (hazard ratio, 5.26; $P<0.001)$. Male sex was also a significant risk for longer duration (hazard ratio, 2.38; $P<0.001$ ). However, it remains unclear whether this may reflect sex-specific immune response activity or social behavior. For prevention of disease spread, we need to pay special attention to patients with these characteristics.

Several limitations in our study should be mentioned. The study was based on retrospective observational findings, and the obtained outcomes may have several biases. Thus, unintentional loss of follow-up may underestimate the outcome or affect the accumulated infection rates. In addition, the SEIR model is a simplified compartmental model and based on assumption of random contact in the compartment and no interaction with outside populations. However, the SEIR model described the basic reproductive number and accumulated infections very accurately, validating the use of this method.

In the real-world setting, infection control measures are installed when an epidemic is recognized. This involves hand washing or social distancing and effectively reduces the reproductive number. Because of this, long-term prediction using computer-based modeling can become inaccurate. However, modeling provides important information at least on short-term prediction as well as on the efficacy of ongoing control measures.

\section{Conclusions}

Our epidemiologic analyses of the HAdV-54 outbreak on the isolated island of Oki Island, Japan, showed that early compartmentalization of symptomatic and asymptomatic individuals without chance of contact is a simple and effective method to control an HAdV-54 outbreak.

Supplementary Information The online version contains supplementary material available at https://doi.org/10.1007/s10384-021-00826-8.

Funding None.

\section{Compliance with Ethical Standards}

Conflict of interests Y. Omatsu, None; D. Miyazaki, Lecture fee (Santen, Senju, Alcon); Y. Shimizu, None; K. Matsuura, None; S. Sasaki, None; Y. Inoue, Grant (Santen, Senju, Alcon); E. Uchio, None; T. Fujimoto, None.

\section{References}

1. Darougar S, Grey RH, Thaker U, McSwiggan DA. Clinical and epidemiological features of adenovirus keratoconjunctivitis in London. Br J Ophthalmol. 1983;67:1-7.

2. Hara J, Okamoto S, Minekawa Y, Yamazaki K, Kase T. Survival and disinfection of adenovirus type 19 and enterovirus 70 in ophthalmic practice. Jpn J Ophthalmol. 1990;34:421-7. 
3. Chintakuntlawar AV, Zhou X, Rajaiya J, Chodosh J. Viral capsid is a pathogen-associated molecular pattern in adenovirus keratitis. PLoS Pathog. 2010;6:e1000841.

4. Aydin Kurna S, Altun A, Oflaz A, Karatay AA. Evaluation of the impact of persistent subepithelial corneal infiltrations on the visual performance and corneal optical quality after epidemic keratoconjunctivitis. Acta Ophthalmol. 2015;93:377-82.

5. Lee CS, Lee AY, Akileswaran L, Stroman D, Najafi-Tagol K, Kleiboeker S, et al. Determinants of outcomes of adenoviral keratoconjunctivitis. Ophthalmology. 2018;125:1344-53.

6. Gonzalez G, Yawata N, Aoki K, Kitaichi N. Challenges in management of epidemic keratoconjunctivitis with emerging recombinant human adenoviruses. J Clin Virol. 2019;112:1-9.

7. Akiyoshi K, Suga T, Fukui K, Taniguchi K, Okabe N, Fujimoto T. Outbreak of epidemic keratoconjunctivitis caused by adenovirus type 54 in a nursery school in Kobe City, Japan in 2008. Jpn J Infect Dis. 2011;64:353-5.

8. Nakamura M, Hirano E, Kowada K, Ishiguro F, Yamagishi Z, Adhikary AK, et al. Surveillance of adenovirus D in patients with epidemic keratoconjunctivitis from Fukui Prefecture, Japan, 1995-2010. J Med Virol. 2012;84:81-6.

9. Uemura T, Migita H, Ueno T, Tsukahara-Kawamura T, Saeki Y, Fujimoto T, et al. Clinical and virological analysis of epidemic keratoconjunctivitis caused by adenovirus type 54 in a regional ophthalmic clinic in Kyushu. Japan Clin Ophthalmol. 2018;12:511-7.

10. 10Hirai Y, Kaita A, Yamamoto S, Kubo H, Iritani N, Ogasawara J. Detection of adenovirus type 54 in patients with epidemic keratoconjunctivitis, 2016-Osaka. Article in Japanese. Infect Agents Surveill Rep. 2018;39:2.

11. 11Anderson RM, May RM. Infectious diseases of humans: dynamics and control. Oxford University Press; 1991.

12. 12Fujimoto T, Hanaoka N, Shimizu H, Matsushima Y, Okabe $\mathrm{N}$, Hagi M, et al. Diagnostic manual for pharyngeal conjunctival fever and epidemic keratoconjunctivitis. 2nd ed. National Institute of Infectious Diseases; 2012.

13. Takeuchi S, Itoh N, Uchio E, Aoki K, Ohno S. Serotyping of adenoviruses on conjunctival scrapings by PCR and sequence analysis. J Clin Microbiol. 1999;37:1839-45.

14. $\mathrm{Xu} \mathrm{W}, \mathrm{McD}$ onough MC, Erdman DD. Species-specific identification of human adenoviruses by a multiplex PCR assay. J Clin Microbiol. 2000;38:4114-20.

15. Fujimoto T, Matsushima Y, Shimizu H, Ishimaru Y, Kano A, Nakajima E, et al. A molecular epidemiologic study of human adenovirus type 8 isolates causing epidemic keratoconjunctivitis in Kawasaki City, Japan in 2011. Jpn J Infect Dis. 2012;65:260-3.

16. Khan A, Naveed M, Dur EAM, Imran M. Estimating the basic reproductive ratio for the Ebola outbreak in Liberia and Sierra Leone. Infect Dis Poverty. 2015;4:13.

17. Gaythorpe KAM, Trotter CL, Lopman B, Steele M, Conlan AJK. Norovirus transmission dynamics: a modelling review. Epidemiol Infect. 2018;146:147-58.

18. Liu Y, Gayle AA, Wilder-Smith A, Rocklöv J. (2020) The reproductive number of COVID-19 is higher compared to SARS coronavirus. J Travel Med. 27:taaa021.

19. Shoman H, Karafillakis E, Rawaf S. The link between the West African Ebola outbreak and health systems in Guinea, Liberia and Sierra Leone: a systematic review. Global Health. 2017;13:1.

20. Glass RI, Parashar UD, Estes MK. Norovirus gastroenteritis. N Engl J Med. 2009;361:1776-85.

21. Estes MK, Prasad BVV, Atmar RL. Noroviruses everywhere: Has something changed? Curr Opin Infect Dis. 2006;19:467-74.

22. Haill CF, Newell P, Ford C, Whitley M, Cox J, Wallis M, et al. Compartmentalization of wards to cohort symptomatic patients at the beginning and end of norovirus outbreaks. J Hosp Infect. 2012;82:30-5.

23. Wiersinga WJ, Rhodes A, Cheng AC, Peacock SJ, Prescott HC. Pathophysiology, transmission, diagnosis, and treatment of coronavirus disease 2019 (COVID-19): a review. JAMA. 2020;324:782-93.

24. Ganyani T, Kremer C, Chen D, Torneri A, Faes C, Wallinga J, et al. Estimating the generation interval for coronavirus disease (COVID-19) based on symptom onset data, March 2020. Euro Surveill. 2020;25:2000257. https://doi.org/10.2807/1560-7917. ES.2020.25.17.2000257.

25. Byambasuren O, Cardona M, Bell K, Clark J, McLaws M-L, Glasziou P. Estimating the extent of asymptomatic COVID-19 and its potential for community transmission: systematic review and meta-analysis. medRxiv. 2020. doi:10.1101.2020.05.10.20097543

Publisher's Note Springer Nature remains neutral with regard to jurisdictional claims in published maps and institutional affiliations. 